\title{
Renormalization constants for Wilson fermion lattice QCD with four dynamical flavours
}

\section{ETM Collaboration}

\author{
Petros Dimopoulos $^{a}$, Roberto Frezzotti ${ }^{b, c}$, Gregorio Herdoiza $^{d}$, Karl Jansen ${ }^{d}$, \\ Vittorio Lubicz ${ }^{e}$, David Palao*c, Giancarlo Rossi ${ }^{b, c}$ \\ a Dipartimento di Fisica, Università di Roma "La Sapienza" \\ Piazzale A. Moro, I-00185 Rome, Italy \\ ${ }^{b}$ Dipartimento di Fisica, Università di Roma "Tor Vergata" \\ Via della Ricerca Scientifica 1, I-00133 Rome, Italy \\ "INFN Sezione di "Roma Tor Vergata" \\ clo Dipartimento di Fisica, Università di Roma "Tor Vergata” \\ Via della Ricerca Scientifica 1, I-00133 Rome, Italy \\ ${ }^{d}$ DESY, Platanenallee 6, D-15738 Zeuthen, Germany \\ e Dipartimento di Fisica, Università di Roma Tre and INFN \\ Via della Vasca Navale 84, I-00146 Rome, Italy \\ E-mail: david.palao@roma2.infn.it
}

\begin{abstract}
We report on an ongoing non-perturbative computation of RI-MOM scheme renormalization constants for the lattice action with four dynamical flavours currently in use by ETMC. For this goal dedicated simulations with four degenerate sea quark flavours are performed at several values of the standard and twisted quark mass parameters. We discuss a method for removing possible O(a) artifacts at all momenta and extrapolating renormalization constant estimators to the chiral limit. We give preliminary results at one lattice spacing.
\end{abstract}

The XXVIII International Symposium on Lattice Field Theory, Lattice2010

June 14-19, 2010

Villasimius, Italy

\footnotetext{
* Speaker.
} 


\section{Introduction}

Simulations including two degenerate light flavours and a non-degenerate doublet of quarks are currently being performed by the European Twisted Mass (ETM) Collaboration. The inclusion of $n_{f}=2+1+1$ flavours is a necessary step to move towards a realistic situation. Fermions are described by the maximally twisted mass lattice QCD (MtmLQCD) action [1] and gluons by the Iwasaki action [2]. While the first physical results are very encouraging [3], dedicated simulations are required to perform the non-perturbative renormalization of operators in a mass-independent scheme, where renormalization constants (RCs) are defined at zero quark mass. In the study of $n_{f}=$ $2+1+1$ QCD ETMC is adopting the RI-MOM scheme [4]. The RCs are evaluated by extrapolating to the chiral limit the RC estimators computed in the theory with $n_{f}=4$ mass degenerate quarks for a range of mass values ${ }^{1}$. Here we report on the progress we made in this project.

\subsection{Action and quark mass parameters}

For the present study we consider the lattice action

$$
S_{L}=S_{I w a}^{\mathrm{YM}}+a^{4} \sum_{x} \sum_{f=1}^{4} \bar{\chi}_{f}\left[\gamma \cdot \widetilde{\nabla}-\frac{a}{2} \nabla^{*} \nabla+m_{0, f}+i \gamma_{5} r_{f} \mu_{f}\right] \chi_{f}(x)
$$

where $\chi_{f}$ is a one-flavour quark field in the so-called twisted basis and in this work $r_{f}$ is set to either 1 or -1 . Passing from the twisted to the physical quark basis ${ }^{2}$

$$
S_{L}=S_{I w a}^{\mathrm{YM}}+a^{4} \sum_{x} \sum_{f=1}^{4} \bar{q}_{f}\left[\gamma \cdot \widetilde{\nabla}-i \gamma_{5} r_{f} e^{i \gamma_{5} r_{f} \theta_{0, f}}\left(-\frac{a}{2} \nabla^{*} \nabla+m_{\mathrm{cr}}\right)+M_{0, f}\right] q_{f}(x) .
$$

The bare mass parameters can be rewritten as

$$
M_{0, f}=\sqrt{\left(m_{0, f}-m_{\mathrm{cr}}\right)^{2}+\mu_{f}^{2}}, \quad \sin \theta_{0, f}=\frac{m_{0, f}-m_{\mathrm{cr}}}{M_{0, f}}, \quad \cos \theta_{0, f}=\frac{\mu_{f}}{M_{0, f}} .
$$

Their renormalized counterparts read $M_{f}=Z_{P} \hat{M}_{f}=\sqrt{Z_{A}^{2} m_{\mathrm{PCAC}}^{2}+\mu_{f}^{2}}$ and $\tan \theta_{f}=\frac{Z_{A} m_{\mathrm{PCAC}}}{\mu_{f}}$. The parametrization in terms of $M$ and $\theta$ is convenient because the leading term of the Symanzik local effective Lagrangian involves only $M$, not $\theta$. As we will see later (see the end of section 2), this remark is at the basis of our method to obtain $O(a)$-improved RC-estimators at all scales even out of maximal twist. Since, for practical reasons, we work in a partially quenched setup with all four flavours having equal mass parameters, we will have to consider in our analysis four quark mass parameters: $M_{\text {sea }}, \theta_{\text {sea }}, M_{\text {val }}, \theta_{\text {val }}$.

\footnotetext{
${ }^{1}$ For Monte Carlo simulations we used a highly optimized implementation of a HMC-like algorithm [5].

${ }^{2}$ The relation between twisted ( $\chi_{f}$ fields) and physical ( $q_{f}$ fields) quark basis is
}

$$
\chi_{f} \rightarrow q_{f}=\exp \left[\frac{i}{2}\left(\frac{\pi}{2}-\theta_{0, f}\right) \gamma_{5} r_{f}\right] \chi_{f}, \quad \bar{\chi}_{f} \rightarrow \bar{q}_{f}=\bar{\chi}_{f} \exp \left[\frac{i}{2}\left(\frac{\pi}{2}-\theta_{0 f}\right) \gamma_{5} r_{f}\right]
$$




\subsection{RI'-MOM scheme and our setup}

The focus of the present study is on flavour non-singlet quark bilinear operators, $O_{\Gamma}=\bar{\chi}_{f} \Gamma \chi_{f^{\prime}}$ (or $\bar{\chi}_{f^{\prime}} \Gamma \chi_{f}$ ), with $\Gamma=S, P, V, A, T$, which are written in terms of $\chi$ and $\bar{\chi}$ quark fields (i.e. in the standard quark basis for untwisted Wilson fermions). RCs are named after the expression of the operators in this basis so as to match the usual notation in the literature about Wilson fermions.

As convenient in lattice studies, we adopt the RI'-MOM scheme [6, 4], which is defined as follows. A first condition fixes the quark field renormalization, namely

$$
Z_{q}^{-1} \frac{-i}{12 N(p)} \sum_{\rho}^{\prime}\left[\frac{\operatorname{Tr}\left(\gamma_{\rho} S_{f}(p)^{-1}\right)}{\tilde{p}_{\rho}}\right]_{\tilde{p}^{2}=\mu^{2}}=1, \quad \text { any } f,
$$

where $\tilde{p}^{2}=\sum_{\mu} \tilde{p}_{\mu}^{2}, \tilde{p}_{\mu} \equiv \frac{1}{a} \sin a p_{\mu}$. The sum $\sum_{\rho}^{\prime}$ only runs over the Lorentz indices for which $p_{\rho}$ is different from zero and $N(p)=\sum_{\rho}^{\prime} 1$. The renormalization condition for the operators $O_{\Gamma}$ reads

$$
Z_{q}^{-1} Z_{\Gamma}^{\left(f f^{\prime}\right)} \operatorname{Tr}\left[\Lambda_{\Gamma}^{\left(f f^{\prime}\right)}(\tilde{p}, \tilde{p}) P_{\Gamma}\right]_{\tilde{p}^{2}=\mu^{2}}=1, \quad f \neq f^{\prime}
$$

Above $S_{f}(p)=a^{4} \sum_{x} e^{-i p x}\left\langle\chi_{f}(x) \bar{\chi}_{f}(0)\right\rangle$ is the $\chi_{f}$ field propagator in momentum space, while

$$
\Lambda_{\Gamma}^{\left(f f^{\prime}\right)}(p, p)=S_{f}^{-1}(p) G_{\Gamma}^{\left(f f^{\prime}\right)}(p, p) S_{f^{\prime}}^{-1}(p)
$$

denotes the quark bilinear vertex that is obtained by "amputating" the Green function

$$
G_{\Gamma}^{\left(f f^{\prime}\right)}(p, p)=a^{8} \sum_{x, y} e^{-i p(x-y)}\left\langle\chi_{f}(x)\left(\bar{\chi}_{f} \Gamma \chi_{f^{\prime}}\right)(0) \bar{\chi}_{f^{\prime}}(y)\right\rangle \quad \Gamma=S, P, V, A, T .
$$

Barring cutoff effects, RCs are independent of $\operatorname{sign}\left(r_{f}\right)$. For practical reasons here we limit ourselves to $r_{f^{\prime}}=-r_{f}$ in evaluating $Z_{\Gamma}$, see eq. (1.5).

\section{Strategy for RCs in the $N_{f}=4$ theory}

In order to extract useful information from simulations performed with twisted mass Wilson fermions one must know the twist angle, $\omega=\frac{\pi}{2}-\theta$, with good precision. The level of precision requested for $\omega$ depends on the observable of interest. In our case, after an exploratory study on a few $16^{3} \times 32$ lattices [7], and some tests near maximal twist on a $24^{3} \times 48$ lattice we have chosen to work out of maximal twist.

Figure 2 illustrates the difficulties of tuning to maximal twist, i.e. setting $m_{\mathrm{PCAC}}$ to zero, in the simulation setup for RC computations, at least if the lattice spacing is not very fine. Specifically, the slope of $m_{\mathrm{PCAC}}$ vs $1 /(2 \kappa)$ in figure 1 (a) suggests that near $m_{\mathrm{PCAC}}=0$ simulations are in a region with a sharpe change of the slope for $m_{\mathrm{PCAC}}$ where it is difficult to extract useful information. On the other hand figure 1(b) gives a more quantitative view of this problem showing results from one simulation close to the critical point (the point closest to $m_{\mathrm{PCAC}}=0$ in figure 1(a)). It appears that due to the long fluctuations a precise measurement of the PCAC mass will require for this case a very large number of Monte Carlo trajectories. In fact, we have observed a similar feature for all the ensembles with $\left|a m_{\mathrm{PCAC}}\right| \lesssim 0.01$ at both $\beta=1.95$ and $\beta=1.90$ (i.e. for $a \geq 0.08 \mathrm{fm}$ ). 
In summary, working at maximal twist for the chosen range of twisted masses (see table 1) would imply a considerable fine tuning work owing to the difficulties in determining $a m_{\text {PCAC }}$ near $a m_{\mathrm{PCAC}}=0$. To alleviate the problem one would need to increase the value of the twisted mass, $\mu_{f}$, and thus $M_{f}$. Instead, working away from maximal twist, one can avoid the metastable region of parameter space and measure the twist angle with good precision. This comes at the price of a moderate increase of the quark mass $M_{f}$ and of a slightly more involved analysis. In our RCestimators cutoff effects linear in $a$ are expected to be small and can anyway be removed with controlled precision by averaging the results obtained for a given $M_{f}$ at opposite values of $\theta_{f}$.

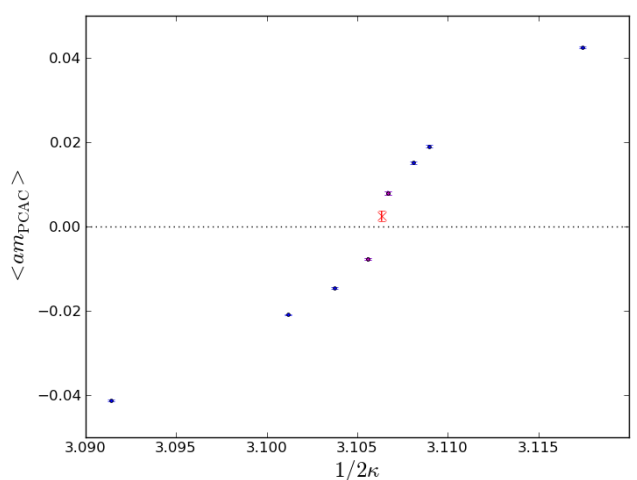

(a)

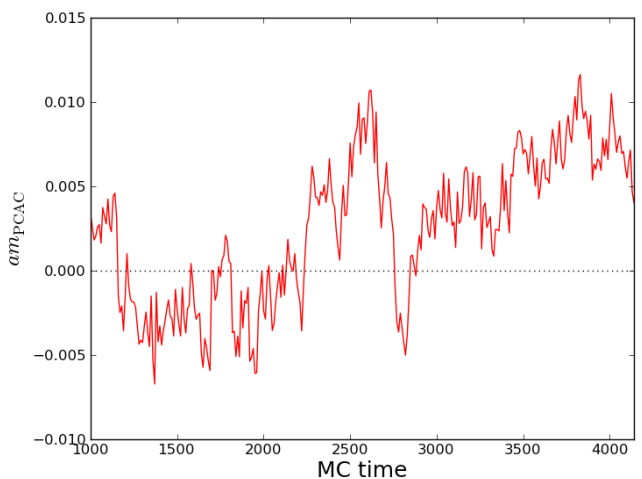

(b)

Figure 1: (a) $a m_{\mathrm{PCAC}}$ versus $1 / 2 \kappa$ on lattices $24^{3} \times 48$ at $\beta=1.95$; (b) Monte Carlo history of $a m_{\mathrm{PCAC}}$ for the most critical case (corresponding to the red cross point) in panel (a).

In fact, from the symmetry of the lattice action $S_{L}$ under $\mathscr{P} \times\left(\theta_{0} \rightarrow-\theta_{0}\right) \times \mathscr{D}_{d} \times\left(M_{0} \rightarrow\right.$ $\left.-M_{0}\right)[1,8,12]$ it follows that the $O\left(a^{2 k+1}\right)$ artifacts occurring in the vacuum expectation values of (multi)local operators $O$ that are invariant under $\mathscr{P} \times\left(\theta_{0} \rightarrow-\theta_{0}\right)$ are quantities that change sign upon changing the sign of $\theta_{0}($ or $\theta)$. Hence $O\left(a^{2 k+1}\right)$ cutoff effects vanish in $\theta$-averages: $\frac{1}{2}\left[\left.\langle O\rangle\right|_{\hat{M}, \theta}+\left.\langle O\rangle\right|_{\hat{M},-\theta}\right]$. The same is true for operator form factors invariant under $\mathscr{P} \times\left(\theta_{0} \rightarrow-\theta_{0}\right)$ and, in particular, for our RC-estimators at all values of $M_{f}$ and $\tilde{p}^{2}$.

\section{Current analysis and preliminary results}

Here we detail the analysis procedure we followed in order to obtain very preliminary results on the RCs of interest. Indeed, at this stage our main goal was checking the feasibility of the project. In particular, the analysis procedure is not yet the optimal one, for instance concerning the order of the various steps, and some refinements, such as the subtraction of the known cutoff effects at $\mathrm{O}\left(a^{2} g^{2}\right)$ [9], are still omitted. While these improvements will be included in the final analysis, the present work shows that the strategy advocated in section 2 allows to extract the RCs of the quark field and quark bilinear operators with $\mathrm{a} \sim 1 \%$ level precision by means of stable simulations at a lattice spacing $(a \sim 0.08 \mathrm{fm})$ which is among the coarsest ones explored in the study of $n_{f}=2+1+1$ QCD by ETMC.

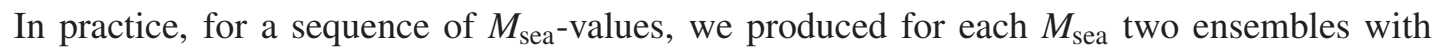
opposite values of $\theta_{\text {sea }}$. We label them as $\mathrm{Ep} / \mathrm{m}$, where $\mathrm{E}=1,2 \ldots$ and $\mathrm{p} / \mathrm{m}$ refers to $\operatorname{sign}\left(\theta_{\text {sea }}\right)$. On 


\begin{tabular}{ccccccc} 
ensemble & $a \mu_{\text {sea }}$ & $a m_{\mathrm{PCAC}}^{\mathrm{sea}}$ & $a M_{0}^{\text {sea }}$ & $\theta^{\text {sea }}$ & $a \mu^{\mathrm{val}}$ & $a m_{\mathrm{PCAC}}^{\mathrm{val}}$ \\
\hline $1 \mathrm{~m}$ & 0.0085 & $-0.04125(13)$ & $0.03288(10)$ & $-1.3093(8)$ & {$[0.0085, \ldots, 0.0298]$} & $-0.0216(2)$ \\
$1 \mathrm{p}$ & 0.0085 & $+0.04249(13)$ & $0.03380(10)$ & $1.3166(7)$ & {$[0.0085, \ldots, 0.0298]$} & $+0.01947(19)$ \\
\hline $3 \mathrm{~m}$ & 0.0180 & $-0.0160(2)$ & $0.02182(9)$ & $-0.601(6)$ & {$[0.0060, \ldots, 0.0298]$} & $-0.0160(2)$ \\
$3 \mathrm{p}$ & 0.0180 & $+0.0163(2)$ & $0.02195(9)$ & $0.610(6)$ & {$[0.0060, \ldots, 0.0298]$} & $+0.0162(2)$ \\
\hline $2 \mathrm{~m}$ & 0.0085 & $-0.02091(16)$ & $0.01821(11)$ & $-1.085(3)$ & {$[0.0085, \ldots, 0.0298]$} & $-0.0213(2)$ \\
$2 \mathrm{p}$ & 0.0085 & $+0.0191(2)$ & $0.01696(16)$ & $1.046(6)$ & {$[0.0085, \ldots, 0.0298]$} & $+0.01909(18)$ \\
\hline $4 \mathrm{~m}$ & 0.0085 & $-0.01459(13)$ & $0.01409(8)$ & $-0.923(4)$ & {$[0.0060, \ldots, 0.0298]$} & $-0.01459(13)$ \\
$4 \mathrm{p}$ & 0.0085 & $+0.0151(2)$ & $0.01441(14)$ & $0.940(7)$ & {$[0.0060, \ldots, 0.0298]$} & $+0.0151(2)$ \\
\hline
\end{tabular}

Table 1: Mass parameters of the ensembles analysed for this contribution. From the formulae in sect. 1.1 it follows that in the valence sector we have $0.013 \lesssim a M^{\mathrm{val}} \lesssim 0.033$ and $0.4 \lesssim\left|\theta_{\mathrm{val}}\right| \lesssim 1.2\left(\theta_{\mathrm{val}} / m_{\mathrm{PCAC}}^{\mathrm{val}}>0\right)$.

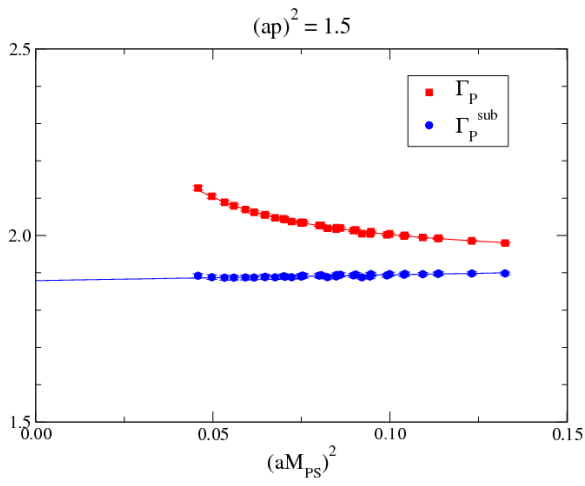

(a)

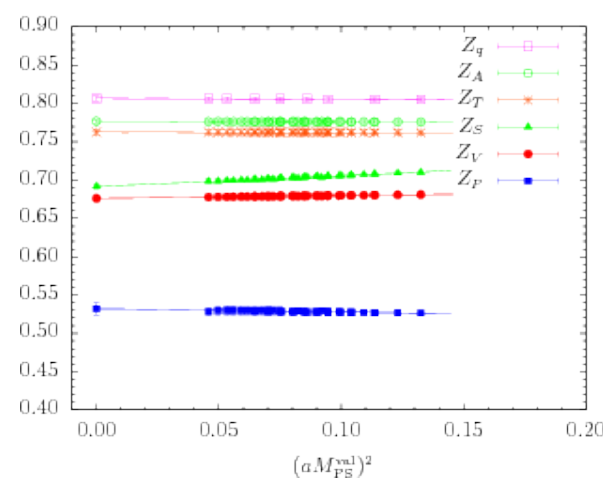

(b)

Figure 2: For the example of ensemble $4 \mathrm{~m},(a \tilde{p})^{2}=1.5$ : (a) subtraction of Goldstone pole contribution and valence chiral extrapolation in $\Gamma_{P}=\operatorname{Tr}\left[\Lambda_{P} P_{P}\right]$; (b) overview of the valence chiral extrapolation for all RCs.

each ensemble Ep/m, with $\left(M_{\mathrm{sea}}^{\mathrm{Ep} / \mathrm{m}}, \theta_{\mathrm{sea}}^{\mathrm{Ep} / \mathrm{m}}\right)$ we compute the RC-estimators for several values of the valence mass parameters $\left(M_{\mathrm{val}}, \theta_{\mathrm{val}}\right)$ and $\tilde{p}^{2}$ (all corresponding to "democratic" momenta $p$, in the sense specified in [10]), as summarized in table 1. Then we proceed in various steps as follows.

Valence chiral limit. A fit of RC-estimators linear in $\left(M_{\mathrm{val}}^{P S}\right)^{2}$ turns out to be numerically adequate (see fig. 2(b)). For $\Gamma=P$ (see fig. 2(a)) or, due to $\mathrm{O}\left(a^{2}\right)$ terms, $\Gamma=S$, we have also kept into account the contribution $\propto\left(M_{\mathrm{val}}^{P S}\right)^{-2}$ coming from the Goldstone boson pole.

$O\left(a^{2} \tilde{p}^{2}\right)$ discretization errors. We applied two different methods, following [10]. In the first method ("M1"), after bringing, via the known [11] perturbative evolution the RC-estimators to a common renormalization scale $\left(\tilde{p}_{\mathrm{M} 1}^{2}=1 / a^{2}\right)$, we remove the remaining $O\left(a^{2} \tilde{p}^{2}\right)$ discretization errors by a linear fit in $\tilde{p}^{2}$. The second method ("M2") consists in simply taking the value of the RCs estimators at a high momentum point fixed in physical units. We chose $\tilde{p}_{\mathrm{M} 2}^{2}=12.2 \mathrm{GeV}^{2}$. The two approaches yield $\mathrm{RC}$ results differing only by cutoff effects. 


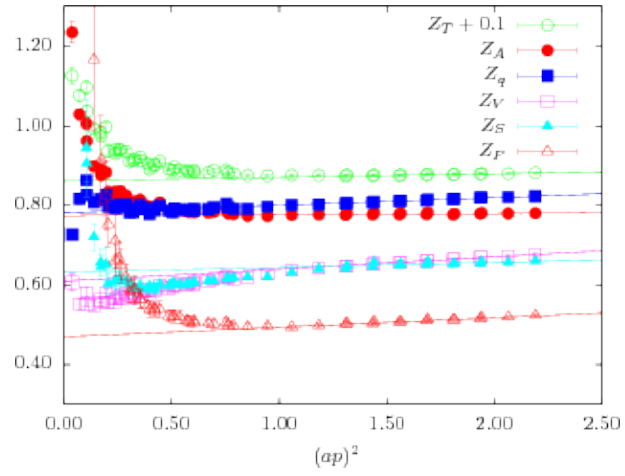

(a)

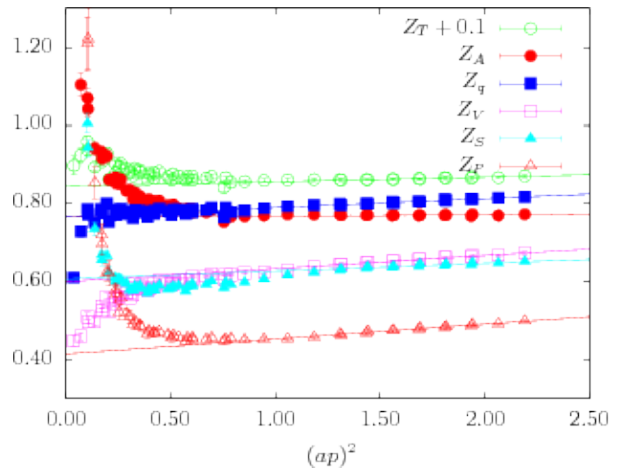

(b)

Figure 3: Residual $\tilde{p}^{2}$-dependence of RC-estimators at scale $1 / a$ and $\mathrm{RC}$ values from method M1 for the cases of ensemble $1 \mathrm{~m}$ (panel (a)) and 2p (panel (b)).

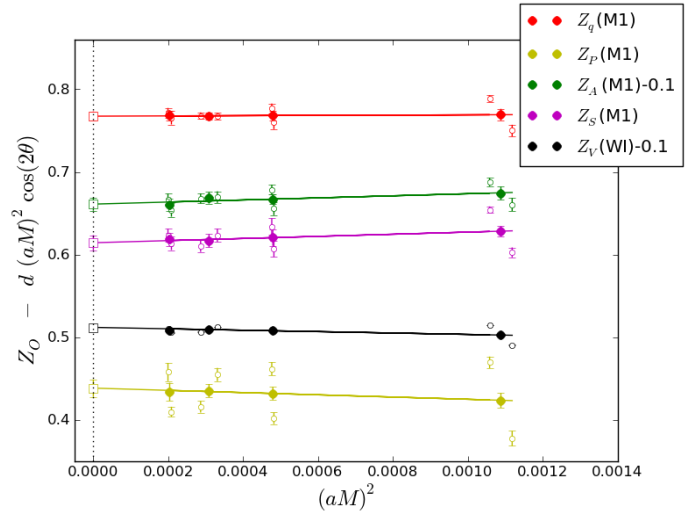

(a)

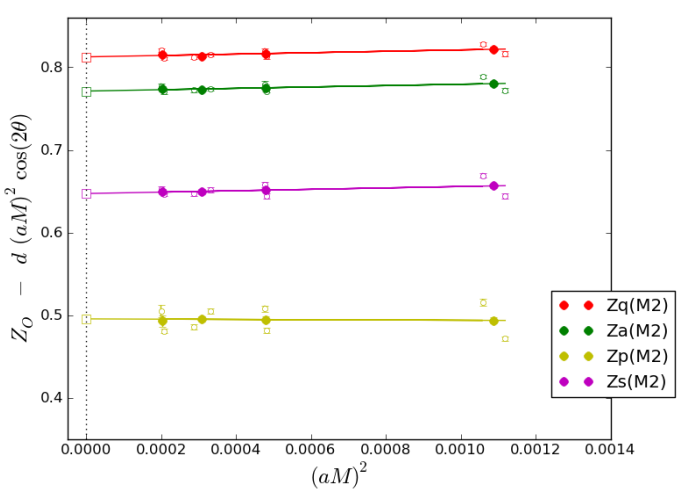

(b)

Figure 4: $M^{\text {sea }}$-dependence before (empty symbols) and after (full symbols) $\theta$-average of RC-estimators for a few operators as well as their chiral limit value. The left (right) panel corresponds to M1 (M2) results. In (a) we also show $Z_{V}(\mathrm{WI})$, which is obtained by exploiting an exact lattice Ward-Takahashi identity (WI).

Removal of $O(a)$ artifacts. It is achieved by $\theta$-average (see section 2) of the RCs estimators,

$$
Z_{\Gamma}\left(M_{\text {sea }}^{\mathrm{E}},\left|\theta_{\text {sea }}^{\mathrm{E}}\right|\right)=\frac{1}{2}\left[\left\langle Z_{\Gamma}\left(\hat{M}_{\text {sea }}^{\mathrm{Ep}}, \theta_{\text {sea }}^{\mathrm{Ep}} ; \theta_{\text {val; } \text { eff }}^{\mathrm{Ep}}\right)\right\rangle+\left\langle Z_{\Gamma}\left(\hat{M}_{\text {sea }}^{\mathrm{Em}}, \theta_{\text {sea }}^{\mathrm{Em}} ; \theta_{\text {val;eff }}^{\mathrm{Em}}\right)\right\rangle\right]
$$

where $\theta_{\text {val;eff }}^{\mathrm{Ep}(\mathrm{m})}$ parameterizes the dominating $\mathrm{O}(a)$ effects in RC-estimators that (in the present analysis) arise from employing $M_{\mathrm{val} ; \mathrm{Ep}(\mathrm{m})}^{\mathrm{PS}}$ in the valence chiral extrapolation.

Sea chiral limit. The quantities $Z_{\Gamma}\left(M_{\text {sea }}^{\mathrm{E}},\left|\theta_{\text {sea }}^{\mathrm{E}}\right|\right)$ are extrapolated to $M_{\text {sea }}=0$ by using the fit Ansatz

$$
Z_{\Gamma}\left(M_{\text {sea }}, \theta_{\text {sea }}\right)=Z_{\Gamma}+A M_{\text {sea }}^{2}+B M_{\text {sea }}^{2} \cos \left(2 \theta_{\text {sea }}\right)
$$

This Ansatz can be justified by an analysis à la Symanzik of the lattice artifacts in $Z_{\Gamma}\left(M_{\text {sea }}, \theta_{\text {sea }}\right)$ up to $\mathrm{O}\left(M_{\text {sea }}^{2}\right)$ and neglecting chiral spontaneous symmetry breaking effects [12].

The first, very preliminary results of this analysis are summarized in table 2. 


\begin{tabular}{cccccccc}
\hline \hline Method & $Z_{A}$ & $Z_{V}$ & $Z_{P}(1 / a)$ & $Z_{S}(1 / a)$ & $Z_{P} / Z_{S}$ & $Z_{T}(1 / a)$ & $Z_{q}(1 / a)$ \\
\hline & & & & & & & \\
M1 & $0.761(08)$ & $0.630(05)$ & $0.438(08)$ & $0.614(09)$ & $0.716(21)$ & $0.753(07)$ & $0.767(06)$ \\
M2 & $0.771(03)$ & $0.674(03)$ & $0.496(04)$ & $0.647(03)$ & $0.767(08)$ & $0.768(03)$ & $0.813(02)$
\end{tabular}

Table 2: Preliminary RC results at $\beta=1.95$ from the analysis of section 3. We also get $Z_{V}(\mathrm{WI})=0.612(1)$.

\section{Conclusions and outlook}

We have described our strategy to compute $\mathrm{O}(a)$ improved operator RCs for the $N_{f}=4$ lattice action currently used by ETMC. We have shown that the method advocated in this work provides very encouraging results at one lattice spacing $(a \sim 0.08 \mathrm{fm})$ that is among the coarsest simulated in the study of QCD with $n_{f}=2+1+1$ dynamical flavours. In particular, the observed dependences of RCs on valence and sea quark masses are mild and quite in line with our experience [10] in $n_{f}=2$ QCD. Besides the technical improvements mentioned in section 3, we plan to possibly add few more ensembles at $a \sim 0.08 \mathrm{fm}(\beta=1.95)$ and to extend our work to other lattice spacings.

We thank IDRIS and INFN/apeNEXT for giving us CPU time necessary for this study.

\section{References}

[1] R. Frezzotti and G. C. Rossi, JHEP 0408 (2004) 007 [arXiv:hep-lat/0306014] and Nucl. Phys. Proc. Suppl. 128 (2004) 193 [arXiv:hep-lat/0311008].

[2] Y. Iwasaki, Nucl. Phys. B 258 (1985) 141.

[3] R. Baron et al. [ETM Collaboration], JHEP 1006 (2010) 111 [arXiv:1004.5284 [hep-lat]] and arXiv:1009.2074 [hep-lat]; F. Farchioni et al. [ETM Collaboration], arXiv:1012.0200 [hep-lat]; G. Herdoiza, plenary talk at the conference Lattice 2010.

[4] G. Martinelli, C. Pittori, C. T. Sachrajda, M. Testa and A. Vladikas, Nucl. Phys. B 445 (1995) 81 [arXiv:hep-lat/9411010].

[5] K. Jansen and C. Urbach, Comput. Phys. Commun. 180 (2009) 2717 [arXiv:0905.3331 [hep-lat]].

[6] E. Franco and V. Lubicz, Nucl. Phys. B 531 (1998) 641 [arXiv:hep-ph/9803491].

[7] A. Deuzeman et al. [ETM Collaboration] PoS LAT2009 (2009) 037.

[8] R. Frezzotti, G. Martinelli, M. Papinutto and G. C. Rossi, JHEP 0604 (2006) 038 [arXiv:hep-lat/0503034].

[9] M. Constantinou, V. Lubicz, H. Panagopoulos and F. Stylianou, JHEP 0910 (2009) 064 [arXiv:0907.0381 [hep-lat]].

[10] M. Constantinou et al. [ETM Collaboration], JHEP 1008 (2010) 068 [arXiv:1004.1115 [hep-lat]].

[11] J. A. Gracey, Nucl. Phys. B 662 (2003) 247 [arXiv:hep-ph/0304113]; K. G. Chetyrkin and A. Retey, Nucl. Phys. B 583 (2000) 3 [arXiv:hep-ph/9910332].

[12] ETM Collaboration, in preparation 